\title{
Coupled Electro-mechanical Behavior of Microtubules
}

\author{
Sundeep Singh ${ }^{1[0000-0002-8342-1622]}$ and Roderick Melnik ${ }^{1,2 *[0000-0002-1560-6684]}$ \\ ${ }^{1}$ MS2Discovery Interdisciplinary Research Institute, Wilfrid Laurier University, 75 University \\ Avenue West, Waterloo, Ontario, Canada, N2L 3C5 \\ ${ }^{2}$ BCAM - Basque Center for Applied Mathematics, Alameda de Mazarredo 14, \\ E-48009 Bilbao, Spain \\ rmelnik@wlu. ca
}

\begin{abstract}
In this contribution, the coupled electro-mechanical behavior of the microtubules has been systematically investigated utilizing a continuum-based finite element framework. A three-dimensional computational model of a microtubule has been developed for predicting the electro-elastic response of the microtubule subjected to external forces. The effects of the magnitude and direction of the applied forces on the mechanics of microtubule have been evaluated. In addition, the effects of variation of microtubule lengths on the electroelastic response subjected to external forces have also been quantified. The results of numerical simulation suggest that the electro-elastic response of microtubule is significantly dependent on both the magnitude and direction of the applied forces. It has been found that the application of shear force results in the attainment of higher displacement and electric potential as compared to the compressive force of the same magnitude. It has been further observed that the output potential is linearly proportional to the predicted displacement and the electric potential within the microtubule. The increase in the length of microtubule significantly enhances the predicted piezoelectric potential under the application of different forces considered in the present study. It is expected that the reported findings would be useful in different avenues of biomedical engineering, such as biocompatible nano-biosensors for health monitoring, drug delivery, noninvasive diagnosis and treatments.
\end{abstract}

Keywords: Electro-mechanical coupling; Microtubules; Piezoelectricity; Computational Modelling

\section{Introduction}

Microtubules are one of the most fundamental structural elements within the cytoskeleton of biological cells that is involved in performing a variety of vital functions, such as providing major mechanical supports and strength to the cells for maintaining and organizing their shapes and facilitating cell division, migration, self-contraction and intracellular transport and signaling [1-3]. Microtubules represent a self-

The reference to the final published version of this paper is: Sundeep Singh \& Roderick Melnik, "Coupled Electro-mechanical Behavior of Microtubules", In: Rojas I., Valenzuela O., Rojas F., Herrera L., Ortuño F. (eds) Bioinformatics and Biomedical Engineering. IWBBIO 2020. Lecture Notes in Computer Science, vol 12108. Springer, Cham (2020). 
assembling polymer composed of heterodimers of $\alpha$-tubulin and $\beta$-tubulin protein that stack head to tail forming the protofilaments. The lateral bonding of the number of protofilaments contributes to the construction of more stable, rigid and structurally intact microtubules in the form a cylindrical surface as shown in Fig. 1 [4]. Thus, microtubules are long hollow cylindrical shaped objects typically made up of 13 protofilaments, but those with 8 to 16 protofilaments have also been reported in the literature [5-6].

Owing to the vital roles played by the microtubules in the biological cells, several studies have been reported in the literature for understanding the mechanics of microtubules in the last two decades [2, 6-12]. The accurate quantification of the mechanical properties and behavior of the microtubules will be extremely important in new biomedical developments, such as in the application of microtubules based novel noninvasive biosensors for disease diagnostics and therapies, and development of advanced biomimetic nanomaterials, e.g., the MT-graphene nanotubes $[1,8]$. Although much of the initial work in this field was confined to experimental and theoretical studies, recently numerical modelling has also been extensively explored for capturing and understanding the mechanical behavior of the microtubules. The numerical modelling approaches are considered to have superiority at capturing the precise information at nano and microscales as compared to experimental studies due to associated limitations and complexities for conducting experiments at such scales [9-10].

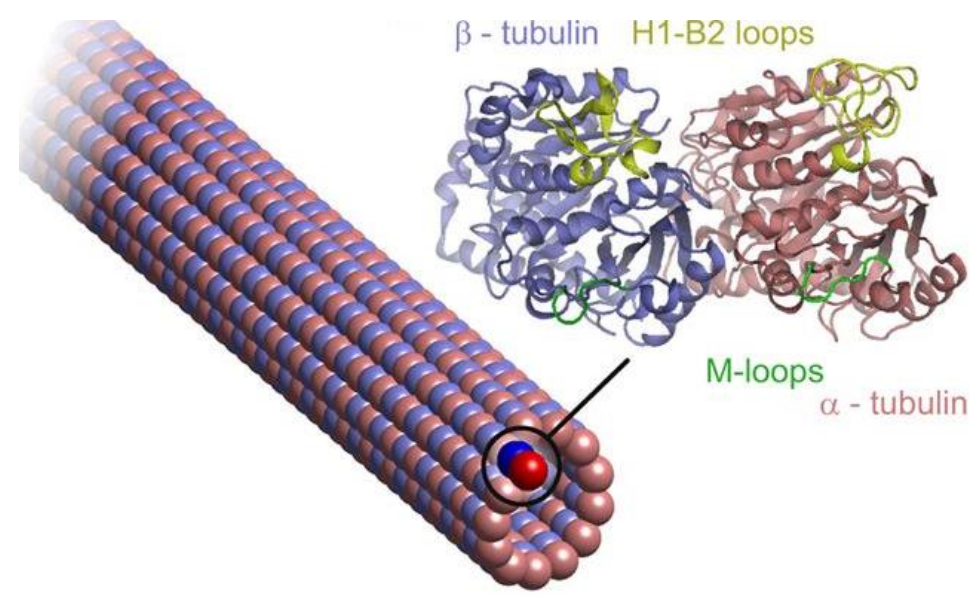

Fig. 1. (Color online). Schematic description of a single microtubule with 13 protofilaments along with the secondary protein structure of an $\alpha-\beta$ tubulin dimer [4]. (This image is reproduced under the terms of the Creative Commons Attribution 4.0 International License, http://creativecommons.org/licenses/by/4.0/, Copyright @ 2019 , Springer Nature Limited).

Most of the numerical studies reported till date are focused on evaluating the mechanical properties of the individual isolated microtubules utilizing only the mechanical coupling between the applied stress and the induced strains. It has also been reported that the microtubules possess piezoelectric properties as a result of electromechanical coupling [13], whereby an electrical charge is produced under the applica- 
tion of mechanical force or vice versa. These studies fall under the research in biomaterials which has received tremendous attention due to its importance in opening the new doors for different promising avenues in biomedical industries, viz., medical devices, wearable sensors, flexible actuators and energy harvesting devices [14]. Thus, the present study focuses on developing a three-dimensional coupled electromechanical model of microtubule for quantifying the piezoelectric behavior under the application of external forces. A parametric analysis has been conducted for evaluating the effects of length of the microtubule, along with the magnitude and direction of the applied forces on the electro-elastic response of the coupled electro-mechanical model.

\section{Continuum Model with Piezoelectricity}

A continuum-based three-dimensional model of individual isolated microtubule considered in the present computational study has been presented in Fig. 2. A $1 \mu \mathrm{m}$ long microtubule with $15 \mathrm{~nm}$ inner diameter and $23 \mathrm{~nm}$ outer diameter has been modelled as a hollow cylindrical tube comprising of 13 protofilaments, as shown in Fig. 2 [15]. The modelling parameters considered in the present computational study are given in Table 1 [16-18].

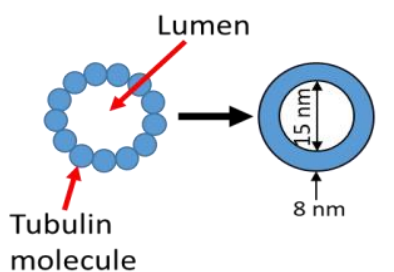

(a)

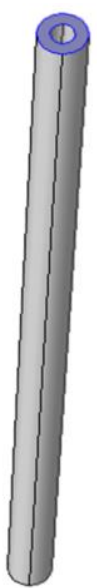

(b)

Fig. 2. (a) Reduction of the 13 protofilament microtubule to continuum-based model, and (b) schematic of single isolated hollow microtubule with $1 \mu \mathrm{m}$ length considered in the present computational study.

The constitutive equations for a linearly coupled electro-mechanical model are given by [19-22]:

$$
\sigma_{i j}=c_{i j k l} \varepsilon_{k l}-e_{i j k} E_{k},
$$




$$
D_{j}=e_{j k l} \varepsilon_{k l}+\kappa_{j k} E_{k},
$$

where $\sigma_{i j}$ are the components of mechanical stress tensor, $\varepsilon_{i j}$ are the component of mechanical strain tensor, $E_{k}$ are the components of electric field vector, $D_{j}$ are the components of electric displacement vector, $c_{i j k l}$ are the linear elastic coefficients, $e_{i j k}$ are the linear piezoelectric coefficients and $\kappa_{i j}$ is the dielectric permittivity coefficients, where subscript $i, j=1,2,3$ and $k, l=1,2,3,4,5,6$. In this computational study, the piezoelectric coefficients of microtubules have been considered similar to that of collagen and have been adapted from [18]. Using Voigt's notation, piezoelectric strain coefficients are given as [18]

$$
d_{i j}=\left[\begin{array}{cccccc}
0 & 0 & 0 & d_{14} & d_{15} & 0 \\
0 & 0 & 0 & d_{15} & -d_{14} & 0 \\
d_{31} & d_{31} & d_{33} & 0 & 0 & 0
\end{array}\right] .
$$

Further, since the present study is formulated using the stress form, thus accordingly the piezoelectric coefficients in strain form given in Eq. (3) have been converted into the stress form utilizing the following relation

$$
e_{i j k}=c_{j k l m} d_{i l m},
$$

where $e_{i j k}$ are the piezoelectric stress coefficients, $c_{j k l m}$ are the components of the elastic tensor and $d_{i l m}$ are the piezoelectric strain coefficients given in Eq. (3).

Table 1. Electro-mechanical characteristics of microtubules considered in the present study.

\begin{tabular}{|c|c|}
\hline $\begin{array}{c}\text { Material } \\
\text { Constants }\end{array}$ & Values \\
\hline \multicolumn{2}{|c|}{ Elastic coefficients (GPa) } \\
\hline $\mathrm{c} 11$ & 2.5577 \\
\hline $\mathrm{c} 22$ & 2.5577 \\
\hline $\mathrm{c} 33$ & 2.5577 \\
\hline $\mathrm{c} 12$ & 1.0962 \\
\hline $\mathrm{c} 13$ & 1.0962 \\
\hline $\mathrm{c} 44$ & 0.7308 \\
\hline $\mathrm{c} 55$ & 0.7308 \\
\hline $\mathrm{c} 66$ & 0.7308 \\
\hline \multicolumn{2}{|c|}{ Relative permittivity } \\
\hline$\kappa$ & 40 \\
\hline Piezoelectric coefficients (pC/N) $^{2}$ & -12 \\
\hline $\mathrm{d}_{14}$ & 6.21 \\
\hline $\mathrm{d}_{15}$ & -4.84 \\
\hline $\mathrm{d}_{31}$ & 0.89 \\
\hline $\mathrm{d}_{33}$ & \\
\hline
\end{tabular}

The relationship between the strain field and displacement field is given by the Cauchy representation 


$$
\varepsilon_{i j}=\frac{1}{2}\left(u_{i, j}+u_{j, i}\right) .
$$

Further, the electric field is related to the gradient of the electric potential as

$$
E_{i}=-\phi,_{i} .
$$

The constitutive equations are further subjected to the equilibrium condition and Gauss's law. Assuming that there is no body forces and no free charges, these governing equations are given by

$$
\begin{gathered}
\sigma_{i j, j}=0, \\
D_{i, i}=0 .
\end{gathered}
$$

The fully coupled electro-mechanical model of microtubule has been numerically implemented using a finite-element method (FEM) based commercial COMSOL Multiphysics 5.2 software [23]. The bottom face of the microtubule has been subjected to fixed and electrically grounded boundary conditions. A compressive and/or shear force has been applied at the top surface of the microtubule and accordingly, the induced stresses, strains, electric field and electric potential have been numerically computed. The initial displacement and electric potential within the computational domain of microtubule have been assumed to be $0 \mathrm{~m}$ and $0 \mathrm{~V}$, respectively. All simulations have been performed in a stationary state regime. An optimum number of heterogeneous tetrahedral mesh elements obtained after conducting a mesh convergence analysis has been used for meshing the computational domain of a hollow cylindrical microtubule using COMSOL's built-in mesh generator. All simulations have been conducted on a Dell T7400 workstation with Quad-core $2.0 \mathrm{GHz}$ Intel ${ }^{\circledR}$ Xeon $^{\circledR}$ processors.

\section{$3 \quad$ Results and Discussion}

In the present computational study, the coupled electro-mechanical model of a microtubule has been modelled by adopting a similar mathematical framework utilized for modeling zinc oxide $(\mathrm{ZnO})$ nanowires [19-20]. Further, the numerical model fidelity and integrity have been evaluated by comparing the predicted results of the current model to those available in the literature, for e.g. [19]. The geometrical details of the nanowire (adapted from [19]) with a hexagonal cross-section considered for numerical validation has been presented in Fig. 3(a). Figure 3(b) presents the piezoelectric potential obtained in the previous study of Hao et al. [19] under the application of 100 $\mathrm{nN}$ compressive force along the $z$-axis at the top surface and the fixed bottom surface of the nanowire, as presented in Fig. 3(a). The piezoelectric potential (in V) obtained under the $100 \mathrm{nN}$ compressive force from the present model utilizing similar geometrical detail and electro-mechanical parameter has been presented in Fig. 3(c). As evident from Fig. 3(c), the piezoelectric potential obtained from the present study com- 
pletely matches with that obtained by [19] (see Fig. 3(b)) in the nanowire with the maximum absolute potential of $0.48 \mathrm{~V}$. The maximum displacement (in $\mathrm{nm}$ ) (refer to Fig. 3(d)) induced at the top surface of the nanowire under the application of $100 \mathrm{nN}$ compressive force from the present model has been found to be $0.03 \mathrm{~nm}$ that is consistent with that obtained in [19]. Moreover, the developed model has also been validated with the applied compressive force of $85 \mathrm{nN}$ at the top surface of the nanowire. Figures 3(e) and 3(f) presents the piezoelectric potential (in V) and displacement (in $\mathrm{nm}$ ) distributions, respectively, obtained from the present model. As evident from Figs. 3(e) and 3(f), the maximum electric potential and displacement have been found to be $0.41 \mathrm{~V}$ and $0.02 \mathrm{~nm}$, respectively, that is in good agreement with those obtained in [19]. Thus, our developed model is consistent with the previous works and lends confidence in the results derived from the developed coupled electro-mechanical model.

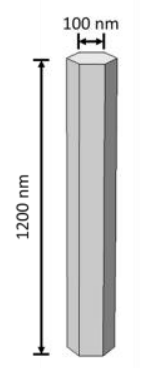

(a)

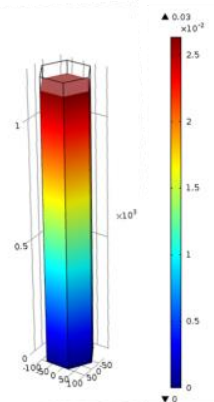

(d)

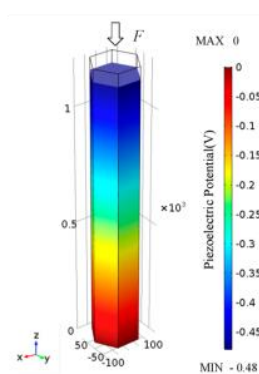

(b)

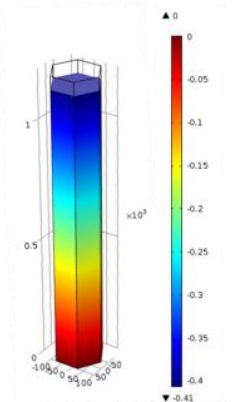

(e)

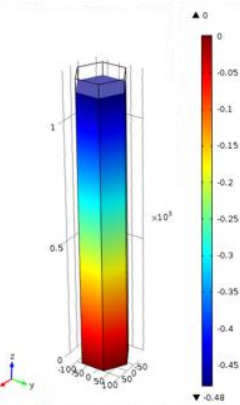

(c)

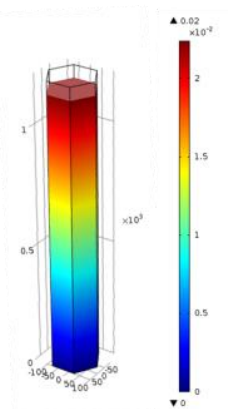

(f)

Fig. 3. (Color online). (a) Schematic illustration of the nanowire having a hexagonal crosssection, (b) piezoelectric potential distribution in the nanowire under a compressive force of $100 \mathrm{nN}$ along the $\mathrm{z}$-axis reported in [19], (c) piezoelectric potential (in V) predicted from the present model of the nanowire under a compressive force of $100 \mathrm{nN}$ along the $\mathrm{z}$-axis, (d) total displacement distribution (in $\mathrm{nm}$ ) predicted from the present model of the nanowire under a compressive force of $100 \mathrm{nN}$ along the z-axis, (e) piezoelectric potential (in V) predicted from the present model of the nanowire under a compressive force of $85 \mathrm{nN}$ along the $\mathrm{z}$-axis, and (f) total displacement distribution (in $\mathrm{nm}$ ) predicted from the present model of the nanowire under a compressive force of $85 \mathrm{nN}$ along the z-axis. (Figure 3(b) has been reproduced from [19] under the terms of the Creative Commons Attribution 4.0 International License, http://creativecommons.org/licenses/by/4.0/, Copyright (C) 1996-2019 MDPI, Basel, Switzerland). 
The results obtained from the coupled electro-mechanical model of a single hollow cylindrical microtubule of $1 \mu \mathrm{m}$ in length under the application of $0.1 \mathrm{nN}$ compressive force uniformly applied at the top surface and fixed bottom surface have been presented in Fig. 4. The displacement distribution within the microtubule under the application of compressive force at the top surface has been presented in Fig. 4(a). As evident from Fig. 4(a), the top surface shows the maximum displacement of $0.09 \mathrm{~nm}$ under the applied force of $0.1 \mathrm{nN}$. It is worthwhile to mention that the displacements induced due to the compressive force lies within the range of that being induced in previous study reported in the literature, for e.g. [16]. In Fig. 4(b) the blue side is the negative potential side and the red side is the ground. As evident from Fig. 4(b), the maximum piezoelectric potential of $0.44 \mathrm{mV}$ has been obtained under the compressive load of $0.1 \mathrm{nN}$. Further, the maximum stress induced in the microtubule under the compressive load has been found to be $0.23 \mathrm{MPa}$, as shown in Fig. 4(c). The displacement, electric potential and stress distributions predicted from the coupled electro-mechanical model under the application of $0.1 \mathrm{nN}$ shear force at the top surface and fixed bottom have been presented in Fig. 5. The maximum displacement and maximum electric potential under the application of $0.1 \mathrm{nN}$ shear force have been found to be $408.86 \mathrm{~nm}$ (see Fig. 5(a)) and $0.62 \mathrm{mV}$ (see Fig. 5(b)), respectively. Accordingly, the maximum stress induced within the hollow microtubule under the application of shear force has been found to be $36.4 \mathrm{MPa}$ and is concentrated at the bottom edges of the microtubule due to significant bending, as shown in Fig. 5(c). Thus, the application of shear force has a far more pronounced effect on the electro-elastic response of a microtubule as compared to the compressive force of the same magnitude.

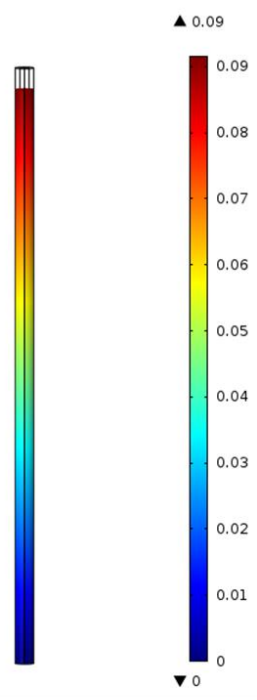

(a)



(b)



(c)

Fig. 4. (Color online). Electro-elastic response of a hollow cylindrical microtubule subjected to a compressive force of $0.1 \mathrm{nN}$ at the top surface: (a) total displacement distribution (in $\mathrm{nm}$ ), (b) electric potential distribution (in $\mathrm{mV}$ ), and (c) stress distribution (in $\mathrm{Pa}$ ). 




(a)

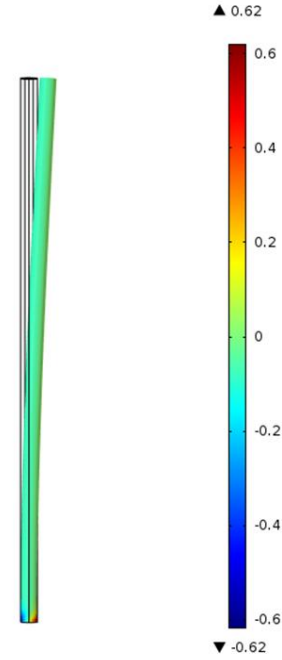

(b)

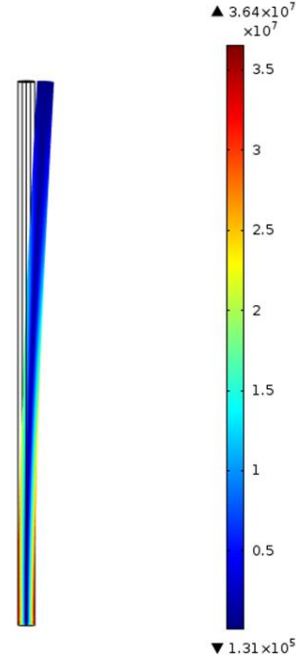

(c)

Fig. 5. (Color online). Electro-elastic response of a hollow cylindrical microtubule subjected to a shear force of $0.1 \mathrm{nN}$ at the top surface: (a) total displacement distribution (in $\mathrm{nm}$ ), (b) electric potential distribution (in $\mathrm{mV}$ ), and (c) stress distribution (in $\mathrm{Pa}$ ).

The effect of direction of $0.1 \mathrm{nN}$ force applied along the $x$-axis, $y$-axis and $z$-axis on the electro-elastic response of a microtubule has been presented in Table 2. In this table, the maximum predicted electric potential and the displacement have been summarized for a different combination of forces. The value of maximum electric potential has been presented in absolute values [20], so as to better compare the electroelastic response of microtubule under different forces. As evident from Table 2, the application of $0.1 \mathrm{nN}$ shear force results in the generation of bigger displacements and corresponding electric potential within the microtubule when compared to a perpendicular compressive force. This can be attributed to the fact that the piezoelectric coefficient in shear direction $\left(\mathrm{e}_{25}\right)$, i.e. along the $\mathrm{y}$-axis, has the highest magnitude and subsequently results in the enhanced coupling between the strain and the electric field, resulting in a higher electric potential generation. Moreover, the maximum values of both the electric potential and displacements increase when the force applied in the $x$ axis was added to that of the $y$-axis. Further addition of the force in the $z$-direction has a trivial impact on the electro-elastic response of the microtubule. Therefore, the predicted results of the coupled electro-mechanical model of a microtubules demonstrate a coupling effect between the three components of the applied forces and the electroelastic response, viz., induced electric potential and displacements. Consequently, this coupling effect should not be ignored for better predicting the behavior of microtubule subjected to external forces. 
Table 2. Maximum displacement and electric potential obtained within a hollow cylindrical microtubule under the application of $0.1 \mathrm{nN}$ force in different directions at the top surface.

\begin{tabular}{|c|c|c|c|c|}
\hline \multicolumn{3}{|c|}{$\begin{array}{c}\text { Applied force } \\
(\mathrm{nN})\end{array}$} & $\begin{array}{c}\text { Displacement } \\
(\mathrm{nm})\end{array}$ & $\begin{array}{c}\text { Electric potential } \\
(\mathrm{mV})\end{array}$ \\
\hline$x$-axis & $y$-axis & $z$-axis & & 0.44 \\
\hline 0 & 0 & 0.1 & 0.09137 & 0.62 \\
\hline 0 & 0.1 & 0 & 408.86 & 0.65 \\
\hline 0.1 & 0 & 0 & 408.86 & 0.88 \\
\hline 0.1 & 0.1 & 0 & 578.21 & 0.65 \\
\hline 0.1 & 0 & 0.1 & 408.86 & 0.62 \\
\hline 0 & 0.1 & 0.1 & 408.86 & 0.89 \\
\hline 0.1 & 0.1 & 0.1 & 578.21 & \\
\hline
\end{tabular}

As mentioned earlier, one of our main motivations for the present computational study is to evaluate the effect of length of microtubule on the electro-elastic response under the application of external forces. Although microtubules can be up to $50 \mu \mathrm{m}$ long, the typical length of microtubules ranges between 0.5 to $10 \mu \mathrm{m}[15,24-25]$. In what follows, we considered three effective lengths of the microtubule, viz., $1 \mu \mathrm{m}, 5$ $\mu \mathrm{m}$ and $10 \mu \mathrm{m}$, for evaluating the impact of microtubule length on their electro-elastic response under the influence of externally applied forces. Notably, the magnitudes of the force considered in our analysis have been varying from $0.02 \mathrm{nN}$ to $0.1 \mathrm{nN}$, with the increment of $0.02 \mathrm{nN}$ and have been adapted from [16]. The total displacements obtained under the application of different magnitude of compressive forces for the microtubule lengths of $1 \mu \mathrm{m}, 5 \mu \mathrm{m}$ and $10 \mu \mathrm{m}$ have been presented in Fig. 6(a). It can be seen from Fig. 6(a) that the total displacement increases linearly as the externally applied force increases from 0.02 to $0.1 \mathrm{nN}$. However, the slope of increment increases with the increase in the microtubule length from 1 to $10 \mu \mathrm{m}$. Similar trends have been observed for the maximum electric potential generation within the different lengths of the microtubule considered in the present study when subjected to the varying magnitude of compressive forces. The effect of varying magnitudes of shear force on the maximum total displacement and the electric potential generated within the microtubule for different active lengths have been presented in Figs. 7(a) and 7(b), respectively. It has been observed that the trends of shear force are somewhat similar to that of compressive force. However, there is a significant variation in the magnitude of the respective values. For example, the maximum magnitude of displacement with compressive force has been found as $0.99 \mathrm{~nm}$ under the application of $0.1 \mathrm{nN}$ force on a $10 \mu \mathrm{m}$ long microtubule, whereas for shear force the predicted value of total displacement considerably rises to $407 \mu \mathrm{m}$. Similarly, the maximum electric potential generated within the $10 \mu \mathrm{m}$ long microtubule under the compressive force of $0.1 \mathrm{nN}$ has been found to be $4.39 \mathrm{mV}$ in comparison to $6.13 \mathrm{mV}$ under the application of shear force. Thus, the length of microtubules significantly influences the electroelastic response as the coupled electro-mechanical model developed in the present study confirms. 


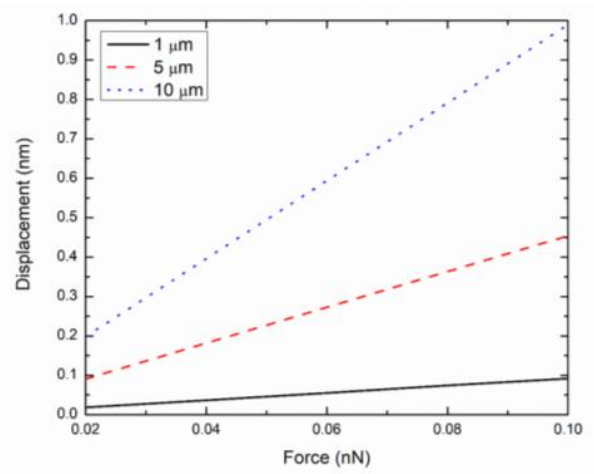

(a)

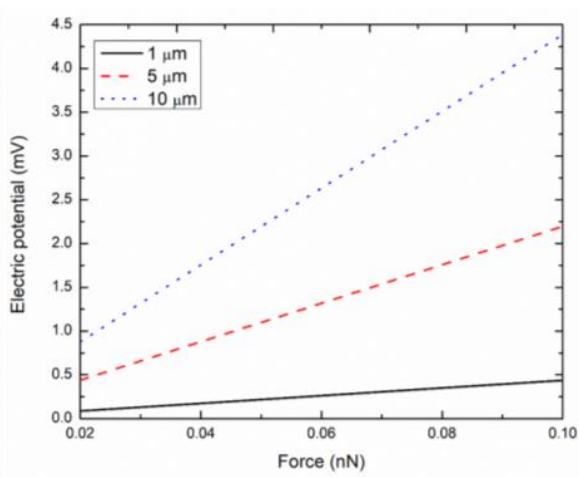

(b)

Fig. 6. Variation of (a) total displacement, and (b) electric potential with the applied compressive force at the top surface of hollow cylindrical microtubule for the active lengths of 1,5 and $10 \mu \mathrm{m}$.

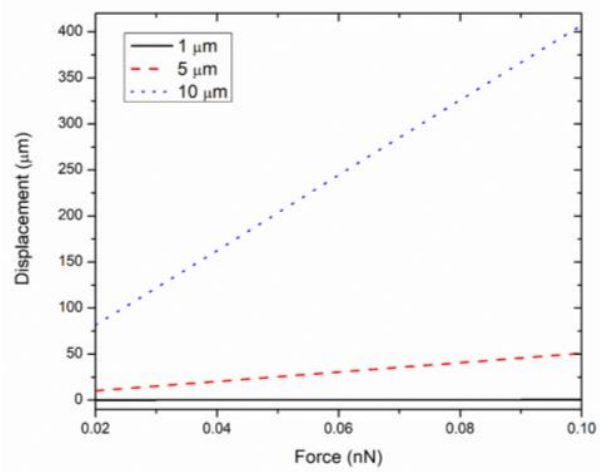

(a)

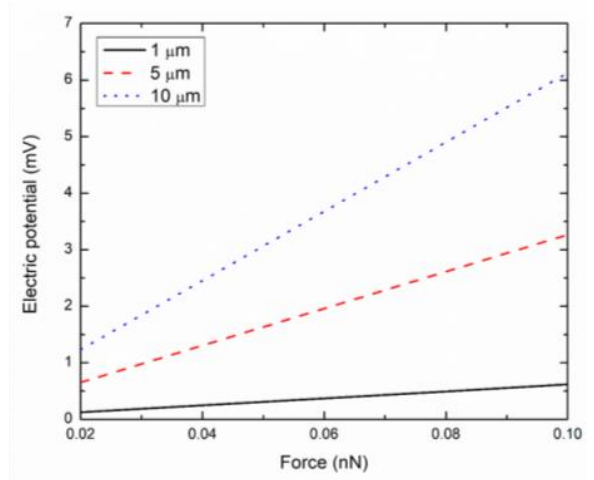

(b)

Fig. 7. Variation of (a) total displacement, and (b) electric potential with the applied shear force at the top surface of hollow cylindrical microtubule for the active lengths of 1,5 and $10 \mu \mathrm{m}$.

\section{Conclusion}

In this work, a coupled electro-mechanical model of a microtubule has been developed for accurately quantifying its complex mechanics at a cellular scale under the influence of external stimuli. Important relations between the applied force and the displacement and applied force and the generated electric potential have been revealed. In particular, it has been observed that the application of shear force leads to both higher displacement and higher electric potential generation as compared to the application of compressive force. Moreover, the increase in the length of microtubules results in a significant rise in the predicted electric potential under the application of 
external forces. This work clearly demonstrates the importance of electro-mechanical coupling in our better understanding of the complex behaviors of microtubules exposed to external forces, which might be difficult to predict utilizing experimental studies. We expect a future extension of the proposed model for biomedical engineering and other related applications ranging from sensing to energy harvesting devices based on biological systems.

\section{Acknowledgments}

Authors are grateful to the NSERC and the CRC Program for their support. RM is also acknowledging the support of the BERC 2018-2021 program and Spanish Ministry of Science, Innovation and Universities through the Agencia Estatal de Investigacion (AEI) BCAM Severo Ochoa excellence accreditation SEV-2017-0718. Authors are also grateful to Prof. Jack Tuszynski as well as to Dr. Jagdish Krishnaswamy for useful discussions, valuable suggestions, and a number of important references.

\section{References}

1. Li, S., Wang, C., Nithiarasu, P.: Simulations on an undamped electromechanical vibration of microtubules in cytosol. Applied Physics Letters 114(25), 253702 (2019).

2. Kučera, O., Havelka, D., Cifra, M.: Vibrations of microtubules: Physics that has not met biology yet. Wave Motion 72, 13-22 (2017).

3. Melnik, R.V.N., Wei, X., Moreno-Hagelsieb, G.: Nonlinear dynamics of cell cycles with stochastic mathematical models. Journal of Biological Systems 17(3), 425-460 (2009).

4. Havelka, D., Deriu, M.A., Cifra, M., Kučera, O.: Deformation pattern in vibrating microtubule: Structural mechanics study based on an atomistic approach. Scientific Reports 7(1), 4227 (2017).

5. Li, S., Wang, C., Nithiarasu, P.: Three-dimensional transverse vibration of microtubules. Journal of Applied Physics 121(23), 234301 (2017).

6. Tuszyński, J.A., Luchko, T., Portet, S., Dixon, J.M.: Anisotropic elastic properties of microtubules. The European Physical Journal E 17(1), 29-35 (2005).

7. Jiang, H., Jiang, L., Posner, J.D., Vogt, B.D.: Atomistic-based continuum constitutive relation for microtubules: elastic modulus prediction. Computational Mechanics 42(4), 607618 (2008).

8. Liew, K.M., Xiang, P., Sun, Y.: A continuum mechanics framework and a constitutive model for predicting the orthotropic elastic properties of microtubules. Composite Structures 93(7), 1809-1818 (2011).

9. Xiang, P., Liew, K.M.: Dynamic behaviors of long and curved microtubules based on an atomistic-continuum model. Computer Methods in Applied Mechanics and Engineering 223, 123-132 (2012).

10. Liew, K.M., Xiang, P., Zhang, L.W.: Mechanical properties and characteristics of microtubules: a review. Composite Structures 123, 98-108 (2015).

11. Marracino, P., Havelka, D., Průša, J., Liberti, M., Tuszynski, J., Ayoub, A.T., Apollonio, F., Cifra, M.: Tubulin response to intense nanosecond-scale electric field in molecular dynamics simulation. Scientific Reports 9(1), 1-14 (2019). 
12. Civalek, Ö., Demir, C.: A simple mathematical model of microtubules surrounded by an elastic matrix by nonlocal finite element method. Applied Mathematics and Computation 289, 335-352 (2016).

13. Tuszynski, J.A., Kurzynski, M.: Introduction to molecular biophysics. CRC Press LLC, Boca Raton (2003).

14. Chae, I., Jeong, C.K., Ounaies, Z., Kim, S. H.: Review on electromechanical coupling properties of biomaterials. ACS Applied Bio Materials 1(4), 936-953 (2018).

15. Thackston, K.A., Deheyn, D.D., Sievenpiper, D. F.: Simulation of electric fields generated from microtubule vibrations. Physical Review E 100(2), 022410 (2019).

16. Katti, D.R., Katti, K.S.: Cancer cell mechanics with altered cytoskeletal behavior and substrate effects: A 3D finite element modeling study. Journal of the Mechanical Behavior of Biomedical Materials 76, 125-134 (2017).

17. Singh, S., Krishnaswamy, J.A., Melnik, R.: Biological cells and coupled electromechanical effects: a new model with nonlocal contributions (Submitted).

18. Denning, D., Kilpatrick, J.I., Fukada, E., Zhang, N., Habelitz, S., Fertala, A., Gilchrist, M.D., Zhang, Y., Tofail, S.A.M., Rodriguez, B.J.: Piezoelectric tensor of collagen fibrils determined at the nanoscale. ACS Biomaterials Science \& Engineering 3(6), 929-935 (2017).

19. Hao, H., Jenkins, K., Huang, X., Xu, Y., Huang, J., Yang, R.: Piezoelectric potential in single-crystalline $\mathrm{ZnO}$ nanohelices based on finite element analysis. Nanomaterials 7(12), 430 (2017).

20. Cardoso, J., Oliveira, F., Proenca, M., Ventura, J.: The influence of shape on the output potential of $\mathrm{ZnO}$ nanostructures: Sensitivity to parallel versus perpendicular forces. Nanomaterials 8(5), 354 (2018).

21. Krishnaswamy, J.A., Buroni, F.C., Garcia-Sanchez, F., Melnik, R., Rodriguez-Tembleque, L., Saez, A.: Lead-free piezocomposites with CNT-modified matrices: Accounting for agglomerations and molecular defects. Composite Structures 224, 111033 (2019).

22. Krishnaswamy, J.A., Buroni, F.C., Garcia-Sanchez, F., Melnik, R., Rodriguez-Tembleque, L., Saez, A.: Improving the performance of lead-free piezoelectric composites by using polycrystalline inclusions and tuning the dielectric matrix environment. Smart Materials and Structures 28, 075032 (2019).

23. COMSOL Multiphysics ${ }^{\circledR}$ v. 5.2. www.comsol.com. COMSOL AB, Stockholm, Sweden.

24. Adnan, A., Qidwai, S., Bagchi, A.: On the atomistic-based continuum viscoelastic constitutive relations for axonal microtubules. Journal of the Mechanical Behavior of Biomedical Materials 86, 375-389 (2018).

25. Xiang, P., Zhang, L.W., Liew, K.M.: Meshfree simulation of temperature effects on the mechanical behaviors of microtubules. Engineering Analysis with Boundary Elements 69, 104-118 (2016). 